\title{
Fast Computation of Pareto Set for Bicriteria Linear Programs with Application to a Diet Formulation Problem
}

\author{
F. Dubeau, M. E. Ntigura Habingabwa \\ Département de Mathématiques, Université de Sherbrooke, Sherbrooke, Canada \\ Email: Francois.Dubeau@USherbrooke.ca, Marie.Emmanuel.Ntigura.Habingabwa@USherbrooke.ca
}

How to cite this paper: Dubeau, F. and
Habingabwa, M.E.N. (2018) Fast Computa-
tion of Pareto Set for Bicriteria Linear Pro-
grams with Application to a Diet Formula-
tion Problem. American Journal of Opera-
tions Research, 8, 323-342.
https://doi.org/10.4236/ajor.2018.85019
Received: July 26, 2018
Accepted: September 2, 2018
Published: September 5, 2018
Copyright $\odot 2018$ by authors and
Scientific Research Publishing Inc.
This work is licensed under the Creative
Commons Attribution International
License (CC BY 4.0).

http://creativecommons.org/licenses/by/4.0/

\begin{abstract}
In case of mathematical programming problems with conflicting criteria, the Pareto set is a useful tool for a decision maker. Based on the geometric properties of the Pareto set for a bicriteria linear programming problem, we present a simple and fast method to compute this set in the criterion space using only an elementary linear program solver. We illustrate the method by solving the pig diet formulation problem which takes into account not only the cost of the diet but also nitrogen or phosphorus excretions.
\end{abstract}

\section{Keywords}

Bicriteria Linear Program, Pareto Set, Criterion Space, Weighted-Sum, Diet Formulation, Taxation System

\section{Introduction}

Animal diet formulation is a very important problem from an economic and environmental point of view, so it is an interesting example in operations research. Many modern animal diet formulation methods tend to take into account nitrogen and phosphorus excretions that are detrimental from an environmental point of view. Following [1], it is appropriate to apply a tax on excretions so as to change the behavior of the producers in the swine industry. These changes in behavior are studied using a formulation of the problem as a bicriteria problem and are obtained by the determination of the Pareto set of the problem. For linear models, this Pareto set is a simple polygonal line. This fact implies that changes in behavior of the producers are abrupt and correspond to particular values of the tax. In other words even in increasing the tax it can 
happen that there is no change in behavior. Behavior changes happend only at very particular values of the tax. We will see that these behaviors correspond to efficient extreme points of the Pareto set, and to every extreme point corresponds a tax interval so that any value of the tax in this interval leads to the behavior given by that extreme point.

The computation and visualization of the Pareto set, also known as the efficiency set, for bicriteria linear programming problems is a useful tool for decision makers. We could try to compute this set in the decision space [2]-[10], but due to the high dimension of this space, it can be a quite large and complicated set. Methods to obtain this set are also complicated, see for example [11]. Fortunately, the geometric aspect of the Pareto set in the criterion (or outcome) space for bicriteria linear program is quite simple [12].

The outline of the paper is the following. The bicriteria problem is presented in Section 2. We will see in Section 3, that the Pareto set of a bicriteria linear problem is a simple polygonal line with $L+1$ extreme points joined by $L$ adjacent segments. Then in Section 4 we presents the link between the geometric structure of the Pareto set and the weighted-sums approach. Then an elementary algorithm to determine the Pareto set in the criterion space is suggested and its complexity is analyzed. Let us point out that this method uses only elementary result from a linear program solver, that is to say the optimal solution (values of the decision variables). This fact is an interesting property of the method.

Few methods exist for computing the Pareto set in the criteria space. One such method is presented in [13]. The method requires information about the dual, assume the feasible set is compact, and determine the Pareto set with at most $2 L$ +4 calls to a linear program solver. Another simple method for bi-criteria problems is presented in [12] to obtain the Pareto set in the criterion space. The algorithm is based on information about the reduced costs of all nonbasic variables, which is equivalent to have information about the solution of the dual problem. For bi-criteria linear problems we could also use a parametric analysis to obtain the Pareto set [11] [14]. The last two methods require that the software used to solve a linear program send information about the dual, reduced cost or postoptimal analysis, which is not always possible for a simple linear program solver. Unfortunately, even if it seems that those two methods require around $2 L$ iterations, their complexities are nowhere analyzed. Moreover they can cycle as explained in [15] (pages 281-282), and [16] (pages 162-166).

Finally, in Section 5, we compute the Pareto sets for least cost diet formulation problems for pig, or any monogastric animal, taking into account the nitrogen and/or phosphorus excretions. Tax systems related to efficient extreme points of this problem are described.

\section{Bicriteria Linear Programming Problem}

Let us consider the standard form of the bicriteria linear programming problem [11] 


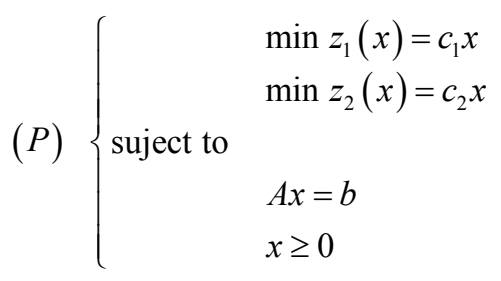

where $x$ is a column vector in $\mathbb{R}^{n}$, and the $c_{k}$ 's $(k=1,2)$ are two row vectors $c_{k}=\left(c_{k, 1}, \cdots, c_{k, n}\right)$ in $\mathbb{R}^{n}$. The feasible set $\mathcal{S}$ in $\mathbb{R}^{n}$ is defined by $\mathcal{S}=\left\{x \in \mathbb{R}^{n} \mid A x=b\right.$ and $\left.x \geq 0\right\}$, where $A$ is a $(m, n)$-matrix, and $b$ is a column vector in $\mathbb{R}^{m}$. Let $C$ be the $(2, n)$-matrix given by

$$
C=\left(\begin{array}{l}
c_{1} \\
c_{2}
\end{array}\right)=\left(\begin{array}{lll}
c_{1,1} & \cdots & c_{1, n} \\
c_{2,1} & \cdots & c_{2, n}
\end{array}\right) .
$$

The feasible set in the criterion space $\mathbb{R}^{2}$ is then $\mathcal{S}_{c}=\left\{z \in \mathbb{R}^{2} \mid z=C x\right.$ for $\left.x \in \mathcal{S}\right\}=C \mathcal{S}$. It is well-known that $\mathcal{S}$ and $\mathcal{S}_{c}$ are polyhedral sets in $\mathbb{R}^{n}$ and $\mathbb{R}^{2}$ respectively. Throughout this paper we will suppose that the two criteria are lower bounded on $\mathcal{S}$ which means that for $i=1,2$ we have

$$
z_{i}^{\min }=\min \left\{z_{i}(x)=c_{i} x \mid x \in \mathcal{S}\right\}>-\infty
$$

\section{Structure of the Pareto Set}

\subsection{Efficiency Set}

A feasible solution $x \in \mathcal{S}$ is an efficient solution if and only if it does not exist any other feasible solution $\bar{x} \in \mathcal{S}$ such that 1) $z_{i}(\bar{x}) \leq z_{i}(x)$ for $i=1,2$, and 2) $z_{j}(\bar{x})<z_{j}(x)$ for at least one $j \in\{1,2\}$. The set of all efficient solutions is called the efficiency set noted $\mathcal{E}$, also called Pareto set. The corresponding set in the criterion space is the set $\mathcal{E}_{c}=C \mathcal{E}$.

\subsection{Geometric Structure}

Under the assumption that the two cost vectors $c_{1}$ and $c_{2}$ are linearly independant, Using weighted-sums, we can replace the bicriteria linear programming problem by a single criterion linear programming problem. We consider $\lambda \in[0,1]$ and the weighted-sum function is

$$
z(x ; \lambda)=(1-\lambda) z_{1}(x)+\lambda z_{2}(x)=\left[(1-\lambda) c_{1}+\lambda c_{2}\right] x,
$$

and we consider the single criteria problem for $\lambda \in[0,1]$

$(P(\lambda)) \begin{cases} & \min z(x ; \lambda)=(1-\lambda) z_{1}(x)+\lambda z_{2}(x)=\left[(1-\lambda) c_{1}+\lambda c_{2}\right] x \\ \text { subject to } & \\ & x \in \mathcal{S} .\end{cases}$

The value function $\varphi(\lambda)$ of $(P(\lambda))$ is defined by

$$
\varphi(\lambda)=\min \{z(x ; \lambda) \mid x \in \mathcal{S}\} .
$$

From [11] we have 


$$
\mathcal{E}=\bigcup_{\lambda \in(0,1)} \arg \min _{x \in \mathcal{S}} z(x ; \lambda) .
$$

Hence the efficiency set $\mathcal{E}$ in the decision space is a connected set and is the union of faces, edges and vertices of $\mathcal{S}$. This set may be quite complex due to the high dimension of the decision space. On the other side $\mathcal{E}_{c}$, which is the image in $\mathbb{R}^{2}$ of $\mathcal{E}$ by a linear transform, is a much simpler set.

Since we have assumed that both criteria are lower bounded on $\mathcal{S}$, it follows that $\mathcal{E}_{c}$ is a simple compact polygonal line. Indeed in that case $\mathcal{E}_{c}$ is the union of a finite number $L$ of segments $\left[Q_{l-1}, Q_{l}\right]$

$$
\mathcal{E}_{c}=\bigcup_{l=1}^{L}\left[Q_{l-1}, Q_{l}\right]
$$

where

$$
\left[Q_{l-1}, Q_{l}\right]=\left\{Q \in \mathbb{R}^{2} \mid Q=(1-\sigma) Q_{l-1}+\sigma Q_{l} \text { for } \sigma \in[0,1]\right\},
$$

and such that

$$
\left(Q_{l-1}, Q_{l}\right) \cap\left(Q_{\tilde{l}-1}, Q_{\tilde{l}}\right)=\varnothing \quad \text { if } l \neq \tilde{l},
$$

with

$$
\left(Q_{l-1}, Q_{l}\right)=\left\{Q \in \mathbb{R}^{2} \mid Q=(1-\sigma) Q_{l-1}+\sigma Q_{l} \text { for } \sigma \in(0,1)\right\} .
$$

To each segment is associated a weight $\lambda_{l-1, l}$ such that the vector $\left(1-\lambda_{l-1, l}, \lambda_{l-1, l}\right)^{t}$ is orthogonal to the segment $\left[Q_{l-1}, Q_{l}\right]$ in $\mathbb{R}^{2}$. To each point $Q$ of $\mathcal{E}_{c}$ is associated an interval $\Lambda(Q)$ defined by

$$
\Lambda(Q)= \begin{cases}{\left[\underline{\lambda}_{l}, \bar{\lambda}_{l}\right]} & \text { if } Q=Q_{l}(l=0, \cdots, L), \\ {\left[\lambda_{l-1, l}, \lambda_{l-1, l}\right]} & \text { if } Q \in\left(Q_{l-1}, Q_{l}\right)(l=1, \cdots, L),\end{cases}
$$

where

$$
\left\{\begin{array}{l}
\underline{\lambda}_{0}=0, \\
\bar{\lambda}_{l-1}=\underline{\lambda}_{l}=\lambda_{l-1, l} \text { for } l=1, \cdots, L, \\
\bar{\lambda}_{L}=1,
\end{array}\right.
$$

with $\bar{\lambda}_{l}-\underline{\lambda}_{l}>0$ for $l=0, \cdots, L$.

\subsection{Weak Efficiency Set}

We will call weak efficiency set, or weak Pareto set, the set defined by

$$
\mathcal{E}^{f}=\bigcup_{\lambda \in[0,1]} \arg \min _{x \in \mathcal{S}} z(x ; \lambda) .
$$

Obviously $\mathcal{E} \subseteq \mathcal{E}^{f}$. In the criteria space we will have $\mathcal{E}_{c}^{f}=C \mathcal{E}^{f}$. Geometrically in the criterion space $\mathbb{R}^{2}$, this means we add to $\mathcal{E}_{c}$ possibly a vertical segment or a ray from $Q_{0}$ in the positive direction of $z_{2}, D_{0}=(0,1)$,

$$
R\left(Q_{0} ; D_{0}\right)=\left\{Q_{0}+\eta D_{0} \mid \eta \in\left(0, \eta_{0}\right]\right\} \subset \mathcal{S}_{c},
$$

and/or a horizontal segment or a ray from $Q_{L}$ in the positive direction of $z_{1}$, $D_{L}=(1,0)$, 


$$
R\left(Q_{L} ; D_{L}\right)=\left\{Q_{L}+\eta D_{L} \mid \eta \in\left(0, \eta_{L}\right]\right\} \subset \mathcal{S}_{c},
$$

where $\eta_{0}$ and $\eta_{L}$ are nonnegative finite or infinite values. They are the maximal values of $\eta$ such that $R\left(Q_{0} ; D_{0}\right)$ and $R\left(Q_{L} ; D_{L}\right)$ are both subsets of $\mathcal{S}_{c}$. To these points on $\mathcal{E}_{c}$ we set

$$
\Lambda(Q)=\left\{\begin{array}{l}
{[0,0] \text { if } Q \in R\left(Q_{0} ; D_{0}\right),} \\
{[1,1] \text { if } Q \in R\left(Q_{L} ; D_{L}\right) .}
\end{array}\right.
$$

\subsection{Link to Parametric Analysis}

The parametric analysis is based on the weighted-sum given by

$$
\tilde{z}(x ; \mu)=z_{1}(x)+\mu z_{2}(x)
$$

for $\mu \in[0,+\infty)$, and the value function in this case is defined by

$$
\tilde{\varphi}(\mu)=\min \{\tilde{z}(x ; \mu) \mid x \in \mathcal{S}\} .
$$

Instead of $(P(\lambda))$, we could consider the single criteria problem for $\mu \geq 0$

$$
(P(\mu)) \begin{cases}\text { subject to } & \min \tilde{z}(x ; \mu)=z_{1}(x)+\mu z_{2}(x)=\left(c_{1}+\mu c_{2}\right) x \\ & x \in \mathcal{S} .\end{cases}
$$

Since $\lambda$ and $\mu$ are related by the formulae

$$
\lambda=\frac{\mu}{1+\mu} \text { and } \mu=\frac{\lambda}{1-\lambda},
$$

to the efficient extreme points $\left\{Q_{l}\right\}_{l=0}^{L}$ on the efficiency set $\mathcal{E}_{c}$ correspond also the following intervals for the parameter $\mu$

$$
\tilde{\Lambda}(Q)= \begin{cases}{\left[\underline{\mu}_{l}, \bar{\mu}_{l}\right]} & \text { if } Q=Q_{l}(l=0, \cdots, L), \\ {\left[\mu_{l-1, l}, \mu_{l-1, l}\right]} & \text { if } Q \in\left(Q_{l-1}, Q_{l}\right)(l=1, \cdots, L),\end{cases}
$$

where

$$
\left\{\begin{array}{l}
\underline{\mu}_{0}=0 \\
\bar{\mu}_{l-1}=\underline{\mu}_{l}=\mu_{l-1, l} \text { for } l=1, \cdots, L \\
\bar{\mu}_{L}=+\infty
\end{array}\right.
$$

In many applications, the parameter $\mu$ is in fact a tax over the the second criteria (for a minimization problem). Interesting enough is to observe that the behavior change (extreme point) only for the critical values $\mu_{l-1, l}$ of the parameter $\mu$. Indeed when $\mu$ increases and its value passes through $\mu_{l-1, l}$, the optimal point, extreme point, move from $Q_{l-1}$ to $Q_{l}$. Thus, any level of taxes $\mu$ strictly between the values $\mu_{l-1, l}=\underline{\mu}_{l}$ and $\mu_{l, l+1}=\bar{\mu}_{l}$ causes the same behavior described by $Q_{l}$.

\section{Computation of the Pareto Set}

\subsection{Preliminaries}

Let us associate to any $Q=\left(z_{1}, z_{2}\right) \in \mathcal{S}_{c}$ the weighted-sum function given by 


$$
\varphi_{Q}(\lambda)=(1-\lambda) z_{1}+\lambda z_{2}
$$

Then the value function $\varphi(\lambda)$ associated to $(P(\lambda))$ is such that

$$
\begin{aligned}
\varphi(\lambda) & =\min \left\{\varphi_{Q}(\lambda) \mid Q \in \mathcal{S}_{c}\right\} \\
& =\min \left\{\varphi_{Q}(\lambda) \mid Q \in \mathcal{E}_{c}\right\} \\
& =\min \left\{\varphi_{Q_{l}}(\lambda) \mid l=0, \cdots, L\right\} .
\end{aligned}
$$

Hence we have the following results.

Theorem 4.1. [12] Let $Q \in \mathcal{E}_{c}$, we have $\varphi(\lambda)=\varphi_{Q}(\lambda)$ if and only if $\lambda \in \Lambda(Q)$.

Theorem 4.2. [12] Let $Q \in \mathcal{E}_{c}$ and $0 \leq \lambda_{1}<\lambda_{2} \leq 1$. Then $\lambda_{1}$ and $\lambda_{2} \in \Lambda(Q)$ if and only if $\left[\lambda_{1}, \lambda_{2}\right] \subseteq \Lambda(Q)$. It follows that $Q$ is one of the $Q_{l}$ $(l \in 0, \cdots, L)$.

Theorem 4.3. [17] The function $\varphi(\lambda)$ is continuous, piecewise linear and concave. The abscissae of slope changes are the increasing values $\lambda_{l-1, l}$ for $l=1, \cdots, L$.

Let us observe that the slope associated to $\varphi_{Q}(\lambda)$ strictly decreases for $Q$ going from $Q_{0}$ to $Q_{L}$ on $\mathcal{E}_{c}$, since $z_{1}$ increases and $z_{2}$ decreases steadily. We deduce the next results.

Theorem 4.4. [12] Let $Q_{i}^{\prime}$ and $Q_{j}^{\prime}$ be two distinct points on $\mathcal{E}_{c}$. For $\lambda \in[0,1],(1-\lambda, \lambda)^{t}$ is orthogonal to the segment $\left[Q_{i}^{\prime}, Q_{j}^{\prime}\right]$ if and only if $\varphi_{Q_{i}^{\prime}}(\lambda)=\varphi_{Q_{j}^{\prime}}(\lambda)$.

Theorem 4.5. Let $Q_{i}^{\prime}$ and $Q_{j}^{\prime}$ be two distinct points on $\mathcal{E}_{c}$ and $\lambda \in[0,1]$, such that $(1-\lambda, \lambda)^{t}$ is orthogonal to the segment $\left[Q_{i}^{\prime}, Q_{j}^{\prime}\right]$. For a fixed $\lambda$, the function $\varphi_{Q}(\lambda)$ is constant as a function of $Q$ on the segment $\left[Q_{i}^{\prime}, Q_{j}^{\prime}\right]$. Let us note this constant value by $\varphi_{Q_{i}^{\prime} Q_{j}^{\prime}}$. Moreover

1) if $\varphi(\lambda)=\varphi_{Q_{i}^{\prime} Q_{j}^{\prime}}$ then $\left[Q_{i}^{\prime}, Q_{j}^{\prime}\right] \subset \mathcal{E}_{c}, \lambda \in \Lambda\left(Q_{i}^{\prime}\right)$ and $\lambda \in \Lambda\left(Q_{j}^{\prime}\right)$;

2) if $\varphi(\lambda)>\varphi_{Q_{i}^{\prime} Q_{j}^{\prime}}$ then $\left(Q_{i}^{\prime}, Q_{j}^{\prime}\right) \cap \mathcal{E}_{c}=\varnothing$.

Theorem 4.6. Let $Q_{i}^{\prime}$ and $Q_{j}^{\prime}$ be two distinct points on $\mathcal{E}_{c}$. If $\lambda \in \Lambda\left(Q_{i}^{\prime}\right)$ is such that $\varphi(\lambda)=\varphi_{Q_{j}^{\prime}}(\lambda)$ then $\lambda \in \Lambda\left(Q_{j}^{\prime}\right)$. Moreover there exists $l \in\{0, \cdots, L\}$ such that $\lambda=\lambda_{l-1, l}$ and $\left[Q_{i}^{\prime}, Q_{j}^{\prime}\right] \subseteq\left[Q_{l-1}, Q_{l}\right] \subseteq \mathcal{E}_{c}$.

Theorem 4.7. Let $Q_{i}^{\prime}$ and $Q_{j}^{\prime}$ be two distinct points on $\mathcal{E}_{c}^{f}$. Let $\lambda_{i}^{\prime} \in \Lambda\left(Q_{i}^{\prime}\right)$ and $\lambda_{j}^{\prime} \in \Lambda\left(Q_{j}^{\prime}\right)$, and consider the following two lines

$$
\mathcal{L}_{i}\left(\lambda_{i}^{\prime}\right)=\left\{Q \in \mathbb{R}^{2} \mid \varphi_{Q}\left(\lambda_{i}^{\prime}\right)=\varphi\left(\lambda_{i}^{\prime}\right)\right\}
$$

and

$$
\mathcal{L}_{j}\left(\lambda_{j}^{\prime}\right)=\left\{Q \in \mathbb{R}^{2} \mid \varphi_{Q}\left(\lambda_{j}^{\prime}\right)=\varphi\left(\lambda_{j}^{\prime}\right)\right\} .
$$

(A) If $\lambda_{i}^{\prime} \neq \lambda_{j}^{\prime}$, the point of intersection of $\mathcal{L}_{i}\left(\lambda_{i}^{\prime}\right)$ and $\mathcal{L}_{j}\left(\lambda_{j}^{\prime}\right)$ is $\tilde{Q}\left(\lambda_{i}^{\prime}, \lambda_{j}^{\prime}\right)=\left(\psi\left(0 ; \lambda_{i}^{\prime}, \lambda_{j}^{\prime}\right), \psi\left(1 ; \lambda_{i}^{\prime}, \lambda_{j}^{\prime}\right)\right)$ where

$$
\psi\left(\lambda ; \lambda_{i}^{\prime}, \lambda_{j}^{\prime}\right)=\frac{\lambda_{j}^{\prime}-\lambda}{\lambda_{j}^{\prime}-\lambda_{i}^{\prime}} \varphi\left(\lambda_{i}^{\prime}\right)+\frac{\lambda-\lambda_{i}^{\prime}}{\lambda_{j}^{\prime}-\lambda_{i}^{\prime}} \varphi\left(\lambda_{j}^{\prime}\right),
$$

so 


$$
\psi\left(0 ; \lambda_{i}^{\prime}, \lambda_{j}^{\prime}\right)=\frac{\lambda_{j}^{\prime} \varphi\left(\lambda_{i}^{\prime}\right)}{\lambda_{j}^{\prime}-\lambda_{i}^{\prime}}-\frac{\lambda_{i}^{\prime} \varphi\left(\lambda_{j}^{\prime}\right)}{\lambda_{j}^{\prime}-\lambda_{i}^{\prime}}
$$

and

$$
\psi\left(1 ; \lambda_{i}^{\prime}, \lambda_{j}^{\prime}\right)=\frac{\lambda_{j}^{\prime}-1}{\lambda_{j}-\lambda_{i}^{\prime}} \varphi\left(\lambda_{i}^{\prime}\right)+\frac{1-\lambda_{i}^{\prime}}{\lambda_{j}^{\prime}-\lambda_{i}^{\prime}} \varphi\left(\lambda_{j}^{\prime}\right) .
$$

(B) If $\lambda_{i}^{\prime}=\lambda_{j}^{\prime}$, then $\mathcal{L}_{i}\left(\lambda_{i}^{\prime}\right)=\mathcal{L}_{j}\left(\lambda_{j}^{\prime}\right)$ which contains the segment $\left[Q_{i}^{\prime}, Q_{j}^{\prime}\right]$.

\subsection{Algorithm}

In this section we consider both criteria upper bounded on $\mathcal{S}$. In the forthcoming algorithm we initialize the process with the two points $Q_{0}$ and $Q_{L}$ on $\mathcal{E}_{c}$. Then we gradually obtain a sequence of points $\left\{Q_{i}^{\prime}\right\}_{i=0}^{I}$ on $\mathcal{E}_{c}$, and a sequence of intervals associated to these points $\left\{\Lambda^{\prime}\left(Q_{i}^{\prime}\right)=\left[\underline{\lambda}_{i}^{\prime}, \bar{\lambda}_{i}^{\prime}\right]\right\}_{i=0}^{I}$ such that $\Lambda^{\prime}\left(Q_{i}^{\prime}\right) \subseteq \Lambda\left(Q_{i}^{\prime}\right)$ and

$$
\varphi(\lambda)=\varphi_{Q_{i}^{\prime}}(\lambda) \text { for all } \lambda \in \Lambda^{\prime}\left(Q_{i}^{\prime}\right) .
$$

At the end of the process $I=L$ and we have $Q_{l}^{\prime}=Q_{l}$ with

$$
\left[\underline{\lambda}_{l}^{\prime}, \bar{\lambda}_{l}^{\prime}\right]=\Lambda^{\prime}\left(Q_{l}^{\prime}\right)=\Lambda\left(Q_{l}\right)=\left[\underline{\lambda}_{l}, \bar{\lambda}_{l}\right]
$$

for $l=0, \cdots, L=I$.

\section{Algorithm (Pareto bicriteria)}

STEP 0. Initialization.

(A) Enter the data of the problem.

(B) Determine $x_{i}^{*}=\arg \min _{x \in \mathcal{S}} z_{i}(x)$ for $i=1,2$ and set $z_{i}^{\min }=z_{i}\left(x^{*}\right)$. For $i, j=1,2$ and $j \neq i$ set $z_{j \mid i}=z_{j}\left(x_{i}^{*}\right)$. We get the initial point $\tilde{Q}_{0}=\left(z_{1}^{\min }, z_{2||}\right)$ which as the same first coordinate as $Q_{0}$, and $\tilde{Q}_{L}=\left(z_{1 \mid 2}, z_{2}^{\min }\right)$ which as the same second coordinate as $Q_{L}$. Those two points might not be on $\mathcal{E}_{c}$, but are on $\mathcal{E}_{c}^{f}$.

(C) Set $Q_{0}^{\prime}:=\tilde{Q}_{0}$ and $\Lambda^{\prime}\left(Q_{0}^{\prime}\right)=\left[\underline{\lambda}_{0}^{\prime}, \bar{\lambda}_{0}^{\prime}\right]:=[0,0]$;

(D) Set $Q_{1}^{\prime}:=\tilde{Q}_{L}$ and $\Lambda^{\prime}\left(Q_{1}^{\prime}\right)=\left[\underline{\lambda}_{1}^{\prime}, \bar{\lambda}_{1}^{\prime}\right]:=[1,1]$;

(E) Set $I:=1$.

STEP 1. As long that there exists an index $i$ such that $\underline{\lambda}_{i}^{\prime}-\bar{\lambda}_{i-1}^{\prime}>0$, select one such index $i$ and do:

(A) Find $\lambda^{*} \in\left[\bar{\lambda}_{i-1}^{\prime}, \underline{\lambda}_{i}^{\prime}\right]$ such that $\varphi_{Q_{i-1}^{\prime} Q_{i}^{\prime}}^{*}:=\varphi_{Q_{i-1}^{\prime}}\left(\lambda^{*}\right)=\varphi_{Q_{i}^{\prime}}\left(\lambda^{*}\right)$, hence $\lambda^{*} \in[0,1]$ such that $\left(1-\lambda^{*}, \lambda^{*}\right)^{t}$ is orthogonal to the segment $\left[Q_{i-1}^{\prime}, Q_{i}^{\prime}\right]$ (see Theorem 4.4);

(B) Solve $\left(P\left(\lambda^{*}\right)\right)$, compute $Q_{\lambda^{*}}:=\left(z_{1}^{\lambda^{*}}, z_{2}^{\lambda^{*}}\right) \in \mathcal{E}_{c}$ with $\varphi\left(\lambda^{*}\right)=\varphi_{Q_{\lambda^{*}}}\left(\lambda^{*}\right)$;

(C) Update the list of points $\left\{Q_{i}^{\prime}\right\}_{i=0}^{I}$ and their intervals $\left\{\Lambda^{\prime}\left(Q_{i}^{\prime}\right)=\left[\underline{\lambda}_{i}^{\prime}, \bar{\lambda}_{i}^{\prime}\right]\right\}_{i=0}^{I}:$

I) Modification of the intervals. If $\varphi\left(\lambda^{*}\right)=\varphi_{Q_{i-1}^{*} Q_{i}^{*}}^{*}$ then all the segment $\left[Q_{i-1}^{\prime}, Q_{i}^{\prime}\right]$ is in $\mathcal{E}_{c}$ (see Theorem 4.5), and $\varphi(\lambda)$ is defined on $\left[\bar{\lambda}_{i-1}^{\prime}, \underline{\lambda}_{i}^{\prime}\right]$ by 
(see Theorems 4.1 and 4.5)

$$
\varphi(\lambda)= \begin{cases}\varphi_{Q_{i-1}^{\prime}}(\lambda) & \text { for } \lambda \in\left[\underline{\lambda}_{i-1}^{\prime}, \lambda^{*}\right] \\ \varphi_{Q_{i}^{\prime}}(\lambda) & \text { for } \lambda \in\left[\lambda^{*}, \bar{\lambda}_{i}^{\prime}\right] .\end{cases}
$$

We modify as follow:

a) for $Q_{i-1}^{\prime}: \Lambda^{\prime}\left(Q_{i-1}^{\prime}\right)=\left[\underline{\lambda}_{i-1}^{\prime}, \bar{\lambda}_{i-1}^{\prime}\right]:=\left[\underline{\lambda}_{i-1}^{\prime}, \lambda^{*}\right]$;

b) for $Q_{i}^{\prime}: \Lambda^{\prime}\left(Q_{i}^{\prime}\right)=\left[\underline{\lambda}_{i}^{\prime}, \bar{\lambda}_{i}^{\prime}\right]:=\left[\lambda^{*}, \underline{\lambda}_{i}^{\prime}\right]$;

In the sequel no more point will be generated on $\left[Q_{i-1}^{\prime}, Q_{i}^{\prime}\right] \subseteq \mathcal{E}_{c}$.

II) Point insertion and interval modification. If $\varphi\left(\lambda^{*}\right)<\varphi_{Q_{i-1}^{\prime} Q_{i}^{\prime}}^{*}$ then $Q_{\lambda^{*}} \notin\left[Q_{i-1}^{\prime}, Q_{i}^{\prime}\right]$, insert the point and modify intervals as follows (see Theorem 4.6):

a) Insert $Q_{\lambda^{*}}$ between $Q_{i-1}^{\prime}$ and $Q_{i}^{\prime}$ in the list with $\Lambda^{\prime}\left(Q_{\lambda^{*}}\right)=\left[\underline{\lambda}^{*}, \bar{\lambda}^{*}\right]:=\left[\lambda^{*}, \lambda^{*}\right]$;

b) Set $I:=I+1$;

c) If $\varphi_{Q_{\lambda^{*}}}\left(\bar{\lambda}_{i-1}^{\prime}\right)=\varphi_{Q_{i-1}^{\prime}}\left(\bar{\lambda}_{i-1}^{\prime}\right)=\varphi\left(\bar{\lambda}_{i-1}^{\prime}\right)$, then $\left[Q_{i-1}^{\prime}, Q_{\lambda^{*}}\right] \subseteq \mathcal{E}_{c}$ and any $\lambda \in\left[\overline{\lambda_{i-1}^{\prime}}, \lambda^{*}\right]$ is in $\Lambda^{\prime}\left(Q_{\lambda^{*}}\right)$, hence we modify $\Lambda^{\prime}\left(Q_{\lambda^{*}}\right)$ by setting $\bar{\lambda}^{*}:=\bar{\lambda}_{i-1}^{\prime}$;

d) If $\varphi_{Q_{\lambda^{*}}}\left(\underline{\lambda}_{i}^{\prime}\right)=\varphi_{Q_{i}^{\prime}}\left(\underline{\lambda}_{i}^{\prime}\right)=\varphi\left(\underline{\lambda}_{i}^{\prime}\right)$, then $\left[Q_{\lambda^{*}}, Q_{i}^{\prime}\right] \subseteq \mathcal{E}_{c}$ and any $\lambda \in\left[\lambda^{*}, \underline{\lambda}_{i}^{\prime}\right]$ is in $\Lambda^{\prime}\left(Q_{\lambda^{*}}\right)$, hence we modify $\Lambda^{\prime}\left(Q_{\lambda^{*}}\right)$ by setting $\bar{\lambda}^{*}:=\underline{\lambda}_{i}^{\prime}$.

STEP 2. For any $i$ such that $\bar{\lambda}_{i}^{\prime}-\underline{\lambda}_{i}^{\prime}=0$, remove $Q_{i}^{\prime}$ from the list and set $I:=I-1$.

STEP 3. End of the process (and $I=L$ ). The output is the list $\left\{Q_{i} ;\left[\underline{\lambda}_{l}, \bar{\lambda}_{l}\right]\right\}_{l=0}^{L}$.

Let us observe that this process use only optimal solutions of $(P(\lambda))$, optimal values of the decision variables, which is easily obtained from any elementary linear program solver.

Remark 4.8. This algorithm produces at each iteration an inner and an outer approximation. The inner approximation is the polygonal line joining the $Q_{i}^{\prime}$ for $i=0, \cdots, I$. The outer approximation is the polygonal line joining the points $Q_{0}^{\prime}, \tilde{Q}\left(\bar{\lambda}_{0}^{\prime}, \underline{\lambda}_{1}^{\prime}\right), Q_{1}^{\prime}, \tilde{Q}\left(\bar{\lambda}_{1}^{\prime}, \underline{\lambda}_{2}^{\prime}\right), Q_{2}^{\prime}, \cdots, Q_{I-2}^{\prime}, \tilde{Q}\left(\bar{\lambda}_{I-2}^{\prime}, \underline{\lambda}_{I-1}^{\prime}\right), Q_{I-1}^{\prime}$, $\tilde{Q}\left(\bar{\lambda}_{I-1}^{\prime}, \underline{\lambda}_{I}^{\prime}\right), Q_{I}^{\prime}$, as long as the $\tilde{Q}\left(\bar{\lambda}_{i-1}^{\prime}, \underline{\lambda}_{i}^{\prime}\right)$ 's are well determined (see Theorem 4.7). At the end of the algorithm the two approximations agree.

\subsection{Complexity}

In this section we are going to determine the maximum number of calls to a linear program solver to completely determine the Pareto set, or equivalently its $L+1$ efficient extreme points $\left\{Q_{l}\right\}_{l=0}^{L}$. The result is given in the last theorem of this section and says that it takes at most $2 L+3$ calls to a linear program solver to generates the $L+1$ extreme points $\left\{Q_{l}\right\}_{l=0}^{L}$.

We will use the following ordering on $\mathcal{E}_{c}^{f}$. For any two distinct points $Q_{i}^{\prime}$ and $Q_{j}^{\prime}$ on $\mathcal{E}_{c}^{f}$, we will say that $Q_{i}^{\prime}$ precedes $Q_{j}^{\prime}$ on $\mathcal{E}_{c}^{f}$, or equivalently that $Q_{j}^{\prime}$ follows $Q_{i}^{\prime}$ on $\mathcal{E}_{c}^{f}$, if moving from on $\mathcal{E}_{c}^{f}$ in the direction from 
$\tilde{Q}_{0}$ to $\tilde{Q}_{L}$ we move from $Q_{i}^{\prime}$ to $Q_{j}^{\prime}$. We will note $Q_{i}^{\prime}<_{\mathcal{E}} Q_{j}^{\prime}$ or equivalently $Q_{j}^{\prime}>_{\mathcal{E}} Q_{i}^{\prime}$.

Theorem 4.9. The algorithm generates at most 3 points on $\left[Q_{l-1}, Q_{l}\right]$ on $\mathcal{E}_{c}$ and two of these points are $Q_{l-1}$ and $Q_{l}$.

Proof. Let us remark that the algorithm will eventually find a point in $\left[Q_{l-1}, Q_{l}\right]$ for any $l=1, \cdots, L$. Let $Q^{1}$ be the first point generated by the algorithm in $\left[Q_{l-1}, Q_{l}\right]$. This first point can be generated at STEP 0 , an initial point, if $\tilde{Q}_{0}=Q_{0} \in\left[Q_{0}, Q_{1}\right]$ for $l=1$ or $\tilde{Q}_{L}=Q_{L} \in\left[Q_{L-1}, Q_{L}\right]$ for $l=L$. Otherwise, it is generated through STEP 1-C-II, with $Q_{i-1}^{\prime}<_{\mathcal{E}} Q_{l-1}$ and $Q_{l}<_{\mathcal{E}} Q_{i}^{\prime}$. Then this point is included in the list, and there are three cases to study:

1) $Q^{1}=Q_{l-1}=Q_{\lambda^{*}}$ for a $\lambda^{*} \in \Lambda\left(Q_{l-1}\right)=\left[\underline{\lambda}_{l-1}=\lambda_{l-2, l-1}, \bar{\lambda}_{l-1}=\lambda_{l-1, l}\right]$ and we have $\Lambda^{\prime}\left(Q^{1}\right)=\left[\lambda^{* *}, \lambda^{*}\right]$ with $\lambda^{* *} \leq \lambda^{*}$. We will have $\lambda^{* *}=\lambda^{*}$, or $\lambda^{* *}=\lambda_{l-2, l-1}$ if the lower bound is modified through STEP 1-C-II-c (if $Q_{i-1}^{\prime} \in\left[Q_{l-2}, Q_{l-1}\right)$ and $\left.\lambda_{l-2, l-1} \in \Lambda^{\prime}\left(Q_{i-1}^{\prime}\right)\right)$.

2) $Q^{1}=Q_{l}=Q_{\lambda^{*}}$ for a $\lambda^{*} \in \Lambda\left(Q_{l}\right)=\left[\underline{\lambda}_{l}=\lambda_{l-1, l}, \bar{\lambda}_{l}=\lambda_{l, l+1}\right]$ and we have $\Lambda^{\prime}\left(Q^{1}\right)=\left[\lambda^{*}, \lambda^{* *}\right]$ with $\lambda^{*} \leq \lambda^{* *}$. We will have $\lambda^{* *}=\lambda^{*}$, or $\lambda^{* *}=\lambda_{l, l+1}$ if the upper bound is modified through STEP 1-C-II-d (if $Q_{i}^{\prime} \in\left(Q_{l}, Q_{l+1}\right]$ and $\left.\lambda_{l, l+1} \in \Lambda^{\prime}\left(Q_{i}^{\prime}\right)\right)$.

3) $Q^{1} \in\left(Q_{l-1}, Q_{l}\right)$ for $\lambda^{*}=\lambda_{l-1, l}$ and we have $\Lambda^{\prime}\left(Q^{1}\right)=\left[\lambda^{*}, \lambda^{*}\right]$.

Let $Q^{2}$ be the second point generated by the algorithm in $\left[Q_{l-1}, Q_{l}\right] . Q^{1}$ must be one of the two points used to generate $Q^{2}$, and hence $\lambda^{*} \neq \lambda_{l-1, l}$. This point $Q^{2}$ is generated through STEP 1-C-II, and it is included in the list. There are two cases to study:

1) $Q_{i-1}^{\prime}<_{\mathcal{E}} Q_{l-1}$ and $Q_{i}^{\prime}=Q^{1} \in\left(Q_{l-1}, Q_{l}\right]$, we will have $\lambda^{*}<\lambda_{l-1, l}$ and $\lambda^{*} \in\left[\underline{\lambda}_{l-1}, \bar{\lambda}_{l-1}=\lambda_{l-1, l}\right) \subset \Lambda\left(Q_{l-1}\right)$. Consequently $Q^{2}=Q_{\lambda^{*}}=Q_{l-1}$ and $\Lambda^{\prime}\left(Q^{2}\right)=\left[\lambda^{* *}, \lambda^{*}\right]$ with $\lambda^{* *}$ modified as in the preceding case. Moreover if $\lambda_{l-1, l} \in \Lambda^{\prime}\left(Q^{1}\right)$ we will modify the upper bound to get $\Lambda^{\prime}\left(Q^{2}\right)=\left[\lambda^{* *}, \lambda_{l-1, l}\right]$.

2) $Q_{i-1}^{\prime}=Q^{1} \in\left[Q_{l-1}, Q_{l}\right)$ and $Q_{i}^{\prime}>_{\mathcal{E}} Q_{l}$, we will have $\lambda^{*}>\lambda_{l-1, l}$ and $\lambda^{*} \in\left(\underline{\lambda}_{l}=\lambda_{l-1, l}, \bar{\lambda}_{l}\right] \subset \Lambda\left(Q_{l}\right)$. Consequently $Q^{2}=Q_{\lambda^{*}}=Q_{l}$ and $\Lambda^{\prime}\left(Q^{2}\right)=\left[\lambda^{*}, \lambda^{* *}\right]$ with $\lambda^{* *}$ modified as in the preceding case. Moreover if $\lambda_{l-1, l} \in \Lambda^{\prime}\left(Q^{1}\right)$ we will modify the lower bound to get $\Lambda^{\prime}\left(Q^{2}\right)=\left[\lambda_{l-1, l}, \lambda^{* *}\right]$.

Two points of $\left[Q_{l-1}, Q_{l}\right]$ are now in the list. We can have a point $Q^{\prime} \in\left(Q_{l-1}, Q_{l}\right)$ with $\Lambda^{\prime}\left(Q^{\prime}\right)=\left[\lambda_{l-1, l}, \lambda_{l-1, l}\right]$ or an extreme point $Q_{l-1}$, with $\Lambda^{\prime}\left(Q_{l-1}\right)=\left[\underline{\lambda}_{l-1}^{\prime}, \lambda_{l-1, l}\right]$, or $Q_{l}$, with $\Lambda^{\prime}\left(Q_{l}\right)=\left[\lambda_{l-1, l}, \overline{\lambda_{l}^{\prime}}\right]$. Otherwise the two points are the extreme points $Q_{l-1}$ and $Q_{l}$. In that case, if $Q_{i-1}^{\prime}=Q_{l-1}$ and $Q_{i}^{\prime}=Q_{l}$, it can happen that $\underline{\lambda}_{i}^{\prime}-\bar{\lambda}_{i-1}^{\prime}=\lambda_{l-1, l}-\lambda_{l-1, l}=0$ and we will have terminated with the interval $\left[Q_{l-1}, Q_{l}\right]$. Otherwise let us note $Q^{3}$ the third point generated in $\left[Q_{l-1}, Q_{l}\right]$. There are two cases to study:

1) We have only one extreme point $Q_{l-1}$, or $Q_{l}$, of the segment in the list and $Q^{\prime} \in\left(Q_{l-1}, Q_{l}\right)$. As in the preceding paragraph, we will introduce it in the list, and depending of the case, by passing through STEP 1-C-II, $Q^{3}=Q_{l-1}$ if $Q_{i-1}^{\prime}<_{\mathcal{E}} Q_{l-1}$ and $Q_{i}^{\prime}=Q^{\prime}$, or $Q^{3}=Q_{l}$ if $Q_{i-1}^{\prime}=Q^{\prime}$ and $Q_{i}^{\prime}>_{\mathcal{E}} Q_{l}$. Moreover, 
we will have respectively $\Lambda^{\prime}\left(Q_{l-1}\right)=\left[\underline{\lambda}_{l-1}^{\prime}, \lambda_{l-1, l}\right] \subseteq \Lambda\left(Q_{l-1}\right)$ or $\Lambda^{\prime}\left(Q_{l}\right)=\left[\lambda_{l-1, l}, \overline{\lambda_{l}^{\prime}}\right] \subseteq \Lambda\left(Q_{l}\right)$.

2) We already have two extreme points $Q_{l-1}$ and $Q_{l}$ in the list. In that case $Q_{i-1}^{\prime}=Q_{l-1}$ and $Q_{i}^{\prime}=Q_{l}$ and we will have $\lambda^{*}=\lambda_{l-1, l}$ and $Q_{\lambda^{*}} \in\left[Q_{l-1}, Q_{l}\right]$. We pass through STEP 1-C-I and we modify the intervals to get $\Lambda^{\prime}\left(Q_{l-1}\right)=\left[\underline{\lambda}_{l-1}^{\prime}, \lambda_{l-1, l}\right] \subseteq \Lambda\left(Q_{l-1}\right)$ et $\Lambda^{\prime}\left(Q_{l}\right)=\left[\lambda_{l-1, l}, \bar{\lambda}_{l}^{\prime}\right] \subseteq \Lambda\left(Q_{l}\right)$. Since $\bar{\lambda}_{l-1}^{\prime}=\lambda_{l-1, l}=\underline{\lambda}_{l}^{\prime}, Q^{3}=Q_{\lambda^{*}}$ is not added to the list.

In the sequel, the algorithm generate no more point on $\left[Q_{l-1}, Q_{l}\right]$ because if we have two points $Q_{i-1}^{\prime}=Q_{l-1}$ and $Q_{i}^{\prime}=Q_{l}$ we have $\underline{\lambda}_{i}^{\prime}-\bar{\lambda}_{i-1}^{\prime}=\lambda_{l-1, l}-\lambda_{l-1, l}=0$, or else, if we have three points, $Q_{i-1}^{\prime}=Q_{l-1}$ and $Q_{i}^{\prime} \in\left(Q_{l-1}, Q_{l}\right)$ and $Q_{i+1}^{\prime}=Q_{l}$ we have $\underline{\lambda}_{i}^{\prime}-\bar{\lambda}_{i-1}^{\prime}=\lambda_{l-1, l}-\lambda_{l-1, l}=0$ and $\underline{\lambda}_{i+1}^{\prime}-\bar{\lambda}_{i}^{\prime}=\lambda_{l-1, l}-\lambda_{l-1, l}=0$.

Theorem 4.10. If $\tilde{Q}_{0}<_{\mathcal{E}} Q_{0}$, respectively $\tilde{Q}_{L}>{ }_{\mathcal{E}} Q_{L}$, then $\tilde{Q}_{0}$, respectively $\tilde{Q}_{L}$, is eventually removed of the list without any supplementary call to the linear program solver.

Proof. When $Q_{0}$ is introduced in the list, there is no supplementary call for $\left[\tilde{Q}_{0}, Q_{0}\right]$. Similarly for the interval $\left[Q_{L}, \tilde{Q}_{L}\right]$ when $Q_{L}$ is introduced in the list. The points $\tilde{Q}_{0}$ and $\tilde{Q}_{L}$ are removed from the list at STEP 2 since $\Lambda^{\prime}\left(\tilde{Q}_{0}\right)=[0,0]$ and $\Lambda^{\prime}\left(\tilde{Q}_{L}\right)=[1,1]$.

Theorem 4.11. The algorithm generates the extreme points $\left\{Q_{l}\right\}_{l=0}^{L}$ of the Pareto set in at most $2 L+3$ calls to a linear program solver.

Proof. The initialization STEP 0 requires 2 calls. For STEP 1, as we generate the $Q_{l}$ for $l=0, \cdots, L$ and possibly one supplementary call for each segment $\left[Q_{l-1}, Q_{l}\right]$ for $l=1, \cdots, L$, there is at most $2 L+1$ calls. Hence the algorithm requires at most $2 L+3$ calls.

\section{A Real World Application: Pig Diet Formulation}

To illustrate our method of computation of the Pareto set we consider the pig diet formulation problem taking into account not only the cost of the diet but also environmental considerations, such as the reduction of nitrogen or phosphorus excretions. One way to analyze this problem is to rewrite the problem as bicriteria problem. Hence the Pareto set indicates the effect of the reduction of excretions, nitrogen or phosphorus, on the cost of the diet. This information is certainly useful for a decision maker which have to choose a diet which decrease the excretions without being too expensive [1]. Even if in thispaper we describe the problem for the swine industry, the method could be applied to any monogastric animal: pig, rabbit, chicken, etc.

\subsection{Classical Model}

The least cost diet problem, introduced in [18], is a classical linear programming problem [19] [20] [21]. A decision variable $x_{j}$ is assigned to each ingredient and represents the amount (in $\mathrm{kg}$ ) of the $f^{\text {th }}$ ingredient per unit weight $(1 \mathrm{~kg})$ of the feed. Together, they form the decision vector $x=\left(x_{j}\right)_{j=1}^{n}$ in our model. The model's objective function is the diet cost. A vector of unit costs $c=\left(c_{j}\right)_{j=1}^{n}$ is 
used, where each $c_{j}$ represents the unit cost of the $f^{\text {th }}$ ingredient (euro/kg or $\$ / \mathrm{kg}$ ). Thus the total cost of a unit of weight $(1 \mathrm{~kg})$ of diet $x=\left(x_{j}\right)_{j=1}^{n}$ is $z=c x=\sum_{j=1}^{n} c_{j} x_{j}$ which must be minimized over the set of feasible diets denoted by $\mathcal{S}$. The classic least cost animal diet formulation model is:

$$
\left(P_{\text {diet }}\right) \begin{cases} & \min z=c x \\ \text { subject to } & \\ & x \in \mathcal{S}=\left\{x \in \mathbb{R}^{n} \mid A x \lesseqgtr b \text { et } x \geq 0\right\} .\end{cases}
$$

The constraints impose some bounds on the quantity of the different ingredients in the diet. For example a unit of feed is produced (a $1 \mathrm{~kg} \mathrm{mix}$ ), expressed by the constraint $\sum_{j=1}^{n} x_{j}=1$. Some ingredients, or combinations of ingredients, can be imposed on the diet. These restrictions give rise to equality constraints $(=)$ or inequality constraints ( $\geq$ or $\leq$ ). More specifically, to satisfy protein requirements, the following constraints are introduced for the $L$ groups of amino acids contained in the ingredients. We set

$$
\sum_{j=1}^{n} a a_{l j}^{d i g} x_{j} \geq b_{l}^{*} \quad(l=1, \cdots, L)
$$

where $a a_{l j}^{d i g}$ represents the amount of digestible amino acid $l$ contained in a unit of ingredient $j$ and $b_{l}^{*}$ is the minimum amount of digestible amino acid $I$ required. Finally, the diet must satisfy the digestible phosphorus requirements $b_{p h}^{*}$ given by

$$
\sum_{j=1}^{n} p h_{j}^{d i g} x_{j} \geq b_{p h}^{*}
$$

where $p h_{j}^{\text {dig }}$ is the amount of digestible phosphorus contained in a unit of ingredient $j$.

\subsection{Modelling of Nitrogen and Phosphorus Excretions}

Nitrogen and phosphorus excretions are directly related to the excess of amounts of protein (amino acids) and phosphorus in the diet. Hence, we have to establish the protein and the phosphorus contents of the diet and take into account the parts that are actually assimilated.

The protein content of a diet $x=\left(x_{j}\right)_{j=1}^{n}$ is $q_{p r} x=\sum_{j=1}^{n} p r_{j} x_{j}$, where $p r_{j}$ is the amount of protein per unit of ingredient $j$. The total excretion of protein $r_{p r}(x)$ is then given by the amount in protein of the diet from which we remove the amount of protein effectively digested given by $\sum_{l=1}^{L} b_{l}^{*}=b_{p r}^{*}$, then

$$
r_{p r}(x)=q_{p r} x-b_{p r}^{*} \text {. }
$$

Hence decreasing the total excretion $r_{p r}(x)$ is equivalent to decrease the protein content $q_{p r} x$ of the diet while maintained fixed the needs $b_{p r}^{*}$ in protein.

As for the nitrogen, the amount of phosphorus of a unit weight diet $x=\left(x_{j}\right)_{j=1}^{n}$ is $q_{p h} x=\sum_{j=1}^{n} p h_{j} x_{j}$, where $p h_{j}$ is the amount of phosphorus per 
unit of ingredient $j$. The amount $b_{p h}^{*}$ is the the amount of phosphorus which is actually digested. In this way the phosphorus excretion $r_{p h}(x)$ is given by the phosphorus content of the diet from which we remove the amount of phosphorus which is actually digested

$$
r_{p h}(x)=q_{p h} x-b_{p h}^{*} .
$$

Hence, decreasing the phosphorus excretion $r_{p h}(x)$ is equivalent to decreasing the phosphorus content $q_{p r} x$ of the diet while maintained fixed the needs $b_{p h}^{*}$ in phosphorus.

\subsection{Data}

The ingredients and their corresponding variables are described in Table 1. Table 2 contains the entire model together with the values of the technical coefficients of the model.

\subsection{Software}

The algorithm was programmed in MATLAB, which includes in its standard library the linear program solver called Linprog. This software can use the simplex method or an interior point method.

\subsection{Two Criteria Models and Results}

At first we analyse the relation between the cost of the diet and the two different excretions (nitrogen and phosphorus). As a curiosity, we also consider the interactions between the two kind of excretions: nitrogen and phosphorus.

Table 1. List of available ingredients.

\begin{tabular}{ccc}
\hline Type & Ingredient & Variable \\
\hline Cereals & Oats & $x_{1}$ \\
& Hard wheat & $x_{2}$ \\
& Corn & $x_{3}$ \\
Oleaginous & Barley & $x_{4}$ \\
& Soybean meal & $x_{5}$ \\
Animal byproducts & Colza meal & $x_{6}$ \\
& Meat and bones meal & $x_{7}$ \\
Minerals & Animal fat & $x_{8}$ \\
& Dicalcique phosphate & $x_{9}$ \\
& Calcium carbonate & $x_{10}$
\end{tabular}




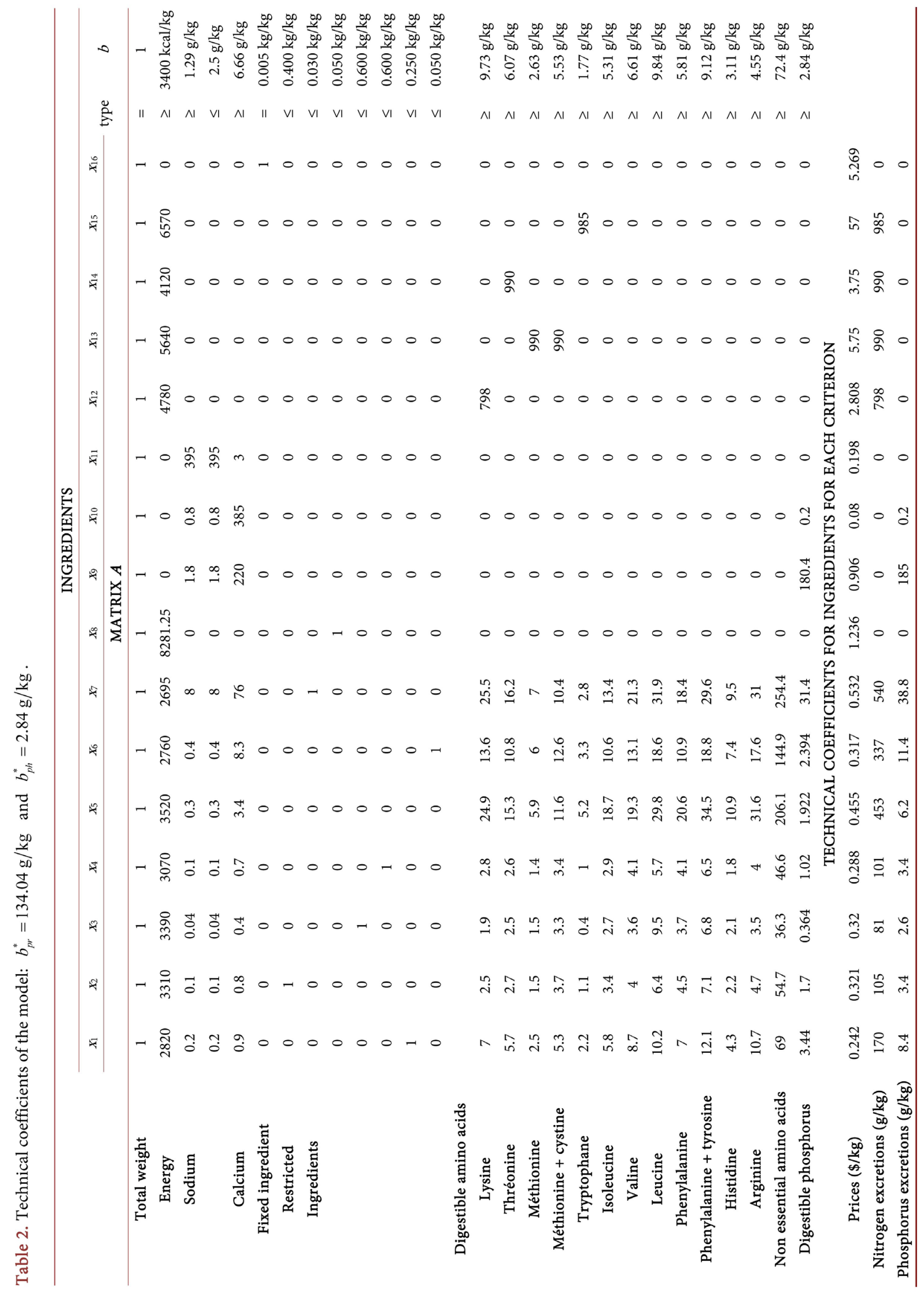




\subsubsection{Cost and Excretions}

We have considered two separate bicriteria models. We look for least cost diets while taking into account the nitrogen excretion for the first model and the phosphorus excretion for the second model. For each of these two bicriteria problems, the Pareto curve indicates the diet cost increase caused by an excretion decrease.

While considering the nitrogen excretion, the problem is :

$$
\left(P_{c, p r}\right) \begin{cases} & \min z_{1}=c x \\ & \min z_{2}=q_{p r} x \\ \text { subject to } & \\ & x \in \mathcal{S} .\end{cases}
$$

Table 3 presents the set of efficient extreme points of the Pareto set in the criterion space, and the Pareto curve is sketched in Figure 1. For this problem, the algorithm detects $L=10$ segments and 11 efficient extreme points

$$
Q_{l}=\left(z_{l, 1}, z_{l, 2}\right)=\left(z_{l, \text { cost }}, z_{l, \text { nitrogen excretion }}\right)
$$

for $l=0, \cdots, L=10$. A total of 22 calls to the linear program solver was required (the predicted maximum is $2 L+3=23$ ).

From its associated weighted-sum model given by

$\left(P_{c, p r}(\lambda)\right) \begin{cases} & \min z(x ; \lambda)=(1-\lambda) z_{1}(x)+\lambda z_{2}(x)=\left[(1-\lambda) c+\lambda q_{p r}\right] x \\ \text { subject to } & \\ & x \in \mathcal{S},\end{cases}$

Table 3. Efficient extreme points in the criterion space $\mathbb{R}^{2}$ and the corresponding taxes

\begin{tabular}{|c|c|c|c|c|c|c|c|}
\hline \multicolumn{6}{|c|}{ Pareto set } & \multicolumn{2}{|c|}{ Taxation system } \\
\hline 1 & $\underline{\lambda}_{l}$ & $\bar{\lambda}_{l}$ & $\begin{array}{l}z_{l, \text { cost }} \\
\$ / \mathrm{kg}\end{array}$ & $\begin{array}{c}z_{l, \text { nitrogen excretion }} \\
\mathrm{g} / \mathrm{kg}\end{array}$ & $\begin{array}{c}z_{l, \text { phosphorus excretion }} \\
\mathrm{g} / \mathrm{kg}\end{array}$ & $\underline{\mu}_{l}$ & $\bar{\mu}_{l}$ \\
\hline 0 & 0 & 0.02617 & 0.40062 & 0.19021 & 6.21226 & 0 & 0.02687 \\
\hline 1 & 0.02617 & 0.13661 & 0.40072 & 0.18661 & 6.18977 & 0.02687 & 0.15823 \\
\hline 2 & 0.13661 & 0.15375 & 0.40147 & 0.18184 & 6.15847 & 0.15823 & 0.18168 \\
\hline 3 & 0.15375 & 0.30996 & 0.40292 & 0.17385 & 6.03610 & 0.18168 & 0.44920 \\
\hline 4 & 0.30996 & 0.49911 & 0.40759 & 0.16347 & 5.67457 & 0.44920 & 0.99643 \\
\hline 5 & 0.49911 & 0.76922 & 0.40816 & 0.16289 & 5.67073 & 0.99643 & 3.33314 \\
\hline 6 & 0.76922 & 0.81451 & 0.40820 & 0.16288 & 5.66990 & 3.33314 & 4.39120 \\
\hline 7 & 0.81451 & 0.81847 & 0.41580 & 0.16115 & 5.44005 & 4.39120 & 4.50866 \\
\hline 8 & 0.81847 & 0.85167 & 0.41608 & 0.16108 & 5.43126 & 4.50866 & 5.74169 \\
\hline 9 & 0.85167 & 0.99010 & 0.41798 & 0.16075 & 5.36141 & 5.74169 & 100.013 \\
\hline 10 & 0.99010 & 1.00000 & 0.42713 & 0.16066 & 5.29463 & 100.013 & $+\infty$ \\
\hline
\end{tabular}
for $(P(c, p r))$. and the corresponding taxes. 


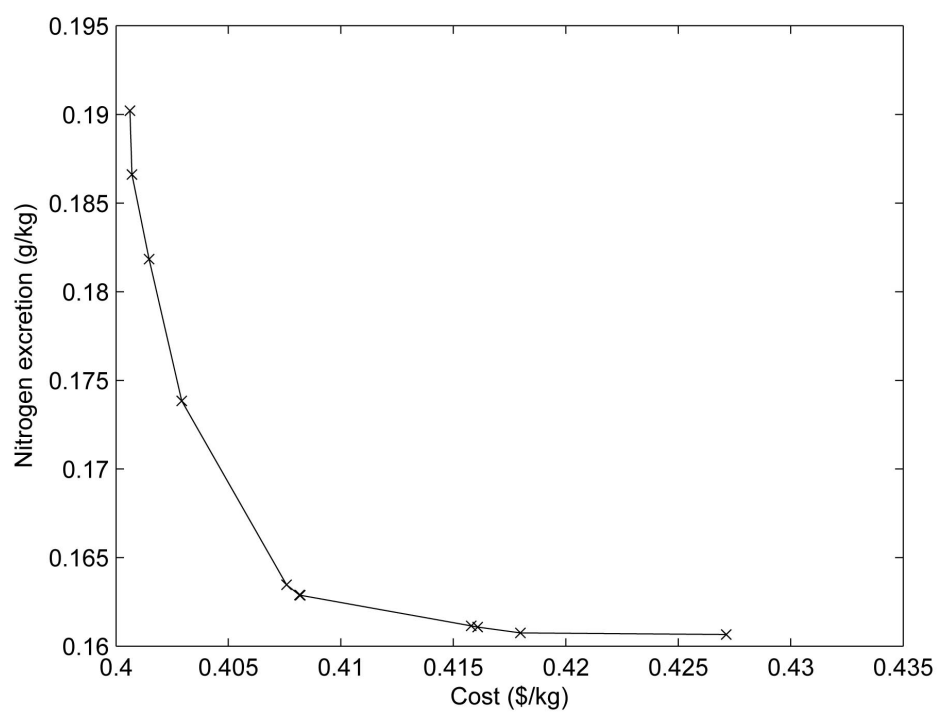

Figure 1. Pareto curve: nitrogen excretion vs diet cost.

we get the following expression for its value function $\varphi(\lambda)$, defined for $\lambda \in[0,1]$, by

$$
\varphi(\lambda)=\min \{z(x ; \lambda) \mid x \in \mathcal{S}\}=(1-\lambda) z_{l, \text { cost }}+\lambda z_{l, \text { nitrogen excretion }}
$$

defined for $\lambda \in\left[\underline{\lambda}_{l}, \bar{\lambda}_{l}\right]$, and $l=0, \cdots, L=10$. So this expression depends on the interval $\left[\underline{\lambda}_{l}, \bar{\lambda}_{l}\right]$ in which $\lambda$ is.

For the parametric model given by

$$
\left(P_{c, p r}(\mu)\right) \begin{cases}\text { subject to } & \min \tilde{z}(x ; \mu)=z_{1}(x)+\mu z_{2}(x)=\left(c+\mu q_{p h}\right) x \\ & x \in \mathcal{S},\end{cases}
$$

we get the following expression for its value function $\tilde{\varphi}(\mu)$ defined for $\mu \in[0,+\infty)$ by

$$
\tilde{\varphi}(\mu)=\min \{\tilde{z}(x ; \mu) \mid x \in \mathcal{S}\}=z_{l, \text { cost }}+\mu z_{l, \text { nitrogen excretion }}
$$

defined for $\mu \in\left[\underline{\mu}_{l}, \bar{\mu}_{l}\right]$, and $l=0, \cdots, L=10$. So this expression depends on the interval $\left[\underline{\mu}_{l}, \bar{\mu}_{l}\right]$ in which $\mu$ is.

So we see that for any tax value in $\left[\mu_{l}, \bar{\mu}_{l}\right]$ we will always have the same expression for the value function $\tilde{\varphi}(\mu)$, or the same behavior given by the efficient extreme point $Q_{l}=\left(z_{l, \text { cost }}, z_{l, \text { nitrogen excretion }}\right)$, and the change in the behavior will happend only when the taxation level $\mu$ passes through the extremities $\underline{\mu}_{l}$ or $\bar{\mu}_{l}$ of this interval

A similar analysis holds for the second bicriteria problem with phosphorus excretion. Indeed, for the phosphorus excretion problem, the model is:

$$
\left(P_{c, p h}\right) \begin{cases} & \min z_{1}=c x \\ & \min z_{3}=q_{p h} x \\ \text { subject to } & \\ & x \in \mathcal{S} .\end{cases}
$$


Table 4 presents the efficient extreme points in the criterion space while the Pareto curve is sketched in Figure 2. For this problem, the algorithm detects $L=22$ segments and 23 extreme points

$$
Q_{l}=\left(z_{l, 1}, z_{l, 3}\right)=\left(z_{l, \text { cost }}, z_{l, \text { phosphorus excretion }}\right)
$$

for $l=0, \cdots, L=22$. A total of 45 calls to the linear program solver was required (the predicted maximum is $2 L+3=47$ ).

From its associated weighted-sum model given by

$$
\left(P_{c, p h}(\lambda)\right) \begin{cases} & \min z(x ; \lambda)=(1-\lambda) z_{1}(x)+\lambda z_{3}(x)=\left[(1-\lambda) c+\lambda q_{p h}\right] x \\ \text { subject to } & \\ & x \in \mathcal{S},\end{cases}
$$

Table 4. Efficient extreme points in the criterion space $\mathbb{R}^{2}$ for $(P(c, p h))$, and the

\begin{tabular}{|c|c|c|c|c|c|c|c|}
\hline \multicolumn{6}{|c|}{ Pareto Set } & \multicolumn{2}{|c|}{ Taxation system } \\
\hline 1 & $\underline{\lambda}_{l}$ & $\bar{\lambda}_{l}$ & $\begin{array}{c}z_{l, \text { cost }} \\
(\$ / \mathrm{kg})\end{array}$ & $\begin{array}{c}z_{l, \text { phosphorus excretion }} \\
(\mathrm{g} / \mathrm{kg})\end{array}$ & $\begin{array}{c}z_{l, \text { nitrogenex cretion }} \\
(\mathrm{g} / \mathrm{kg})\end{array}$ & $\underline{\mu}_{l}$ & $\bar{\mu}_{l}$ \\
\hline 0 & 0 & 0.00428 & 0.40062 & 6.21226 & 0.19021 & 0 & 0.00430 \\
\hline 1 & 0.00428 & 0.00452 & 0.40072 & 6.18977 & 0.18661 & 0.00430 & 0.00454 \\
\hline 2 & 0.00452 & 0.00456 & 0.40164 & 5.98711 & 0.18707 & 0.00454 & 0.00458 \\
\hline 3 & 0.00456 & 0.00500 & 0.40196 & 5.91713 & 0.18927 & 0.00458 & 0.00502 \\
\hline 4 & 0.00500 & 0.00528 & 0.40219 & 5.87162 & 0.18854 & 0.00502 & 0.00531 \\
\hline 5 & 0.00528 & 0.00628 & 0.40310 & 5.69979 & 0.18742 & 0.00531 & 0.00632 \\
\hline 6 & 0.00628 & 0.00708 & 0.40365 & 5.61223 & 0.18802 & 0.00632 & 0.00713 \\
\hline 7 & 0.00708 & 0.00783 & 0.40379 & 5.59297 & 0.18676 & 0.00713 & 0.00789 \\
\hline 8 & 0.00783 & 0.00919 & 0.40400 & 5.56609 & 0.18566 & 0.00789 & 0.00927 \\
\hline 9 & 0.00919 & 0.01003 & 0.40541 & 5.41416 & 0.18737 & 0.00927 & 0.01013 \\
\hline 10 & 0.01003 & 0.01458 & 0.40601 & 5.35505 & 0.18975 & 0.01013 & 0.01479 \\
\hline 11 & 0.01458 & 0.02357 & 0.40633 & 5.33336 & 0.18640 & 0.01479 & 0.02414 \\
\hline 12 & 0.02357 & 0.09694 & 0.40798 & 5.26498 & 0.17596 & 0.02414 & 0.10734 \\
\hline 13 & 0.09694 & 0.11478 & 0.41768 & 5.17458 & 0.16744 & 0.10734 & 0.12967 \\
\hline 14 & 0.11478 & 0.12931 & 0.42351 & 5.12967 & 0.16700 & 0.12967 & 0.14852 \\
\hline 15 & 0.12931 & 0.14182 & 0.42429 & 5.12440 & 0.16609 & 0.14852 & 0.16526 \\
\hline 16 & 0.14182 & 0.48610 & 0.43631 & 5.05165 & 0.16364 & 0.16526 & 0.94589 \\
\hline 17 & 0.48610 & 0.49168 & 0.74777 & 4.72237 & 0.26885 & 0.94589 & 0.96727 \\
\hline 18 & 0.49168 & 0.62773 & 0.79624 & 4.67226 & 0.28486 & 0.96727 & 1.68624 \\
\hline 19 & 0.62773 & 0.69486 & 1.12394 & 4.47793 & 0.38921 & 1.68624 & 2.27723 \\
\hline 20 & 0.69486 & 0.99962 & 1.30843 & 4.39691 & 0.45147 & 2.27723 & 2662.91 \\
\hline 21 & 0.99962 & 0.99998 & 2.06125 & 4.39663 & 0.46634 & 2662.91 & 59645.9 \\
\hline 22 & 0.99998 & 1.00000 & 15.32799 & 4.39641 & 0.42199 & 59645.9 & $+\infty$ \\
\hline
\end{tabular}
corresponding taxes. 


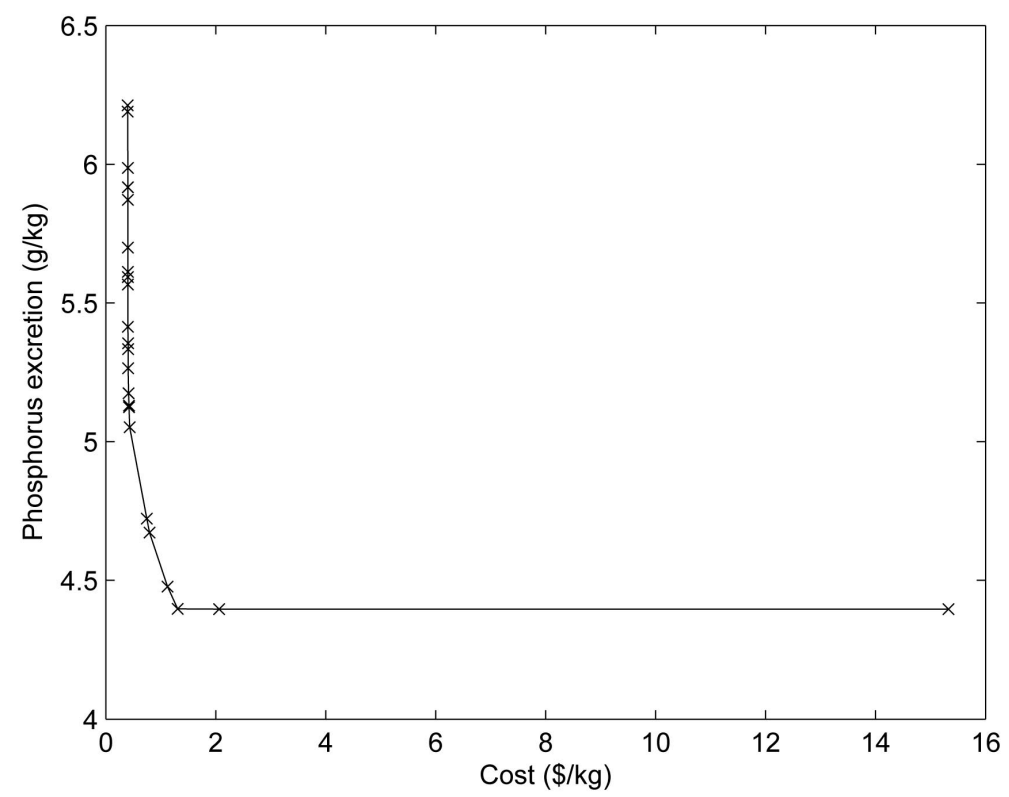

Figure 2. Pareto curve: phosphorus excretion vs diet cost.

we get the following expression for its value function $\varphi(\lambda)$ defined for $\lambda \in[0,1]$ by

$$
\varphi(\lambda)=\min \{z(x ; \lambda) \mid x \in \mathcal{S}\}=(1-\lambda) z_{l, \text { cost }}+\lambda z_{l, \text { phosphorus excretion }}
$$

defined for $\lambda \in\left[\underline{\lambda}_{l}, \bar{\lambda}_{l}\right]$, and $l=0, \cdots, L=22$. So this expression depends on the interval $\left[\underline{\lambda}_{l}, \bar{\lambda}_{l}\right]$ in which $\lambda$ is.

For the parametric model given by

$$
\left(P_{c, p h}(\mu)\right) \begin{cases} & \min \tilde{z}(x ; \mu)=z_{1}(x)+\mu z_{3}(x)=\left(c+\mu q_{p h}\right) x \\ \text { subject to } & \\ & x \in \mathcal{S},\end{cases}
$$

we get the following expression for its value function $\tilde{\varphi}(\mu)$ defined for $\mu \in[0,+\infty)$ by

$$
\tilde{\varphi}(\mu)=\min \{\tilde{z}(x ; \mu) \mid x \in \mathcal{S}\}=z_{l, \text { cost }}+\mu z_{l, \text { phosphorus excretion }}
$$

defined for for $\mu \in\left[\mu_{l}, \bar{\mu}_{l}\right]$, and $l=0, \cdots, L=22$. So this expression depends on the interval $\left[\underline{\mu}_{l}, \bar{\mu}_{l}\right]$ in which $\mu$ is.

So we see that for any tax value in $\left[\mu_{l}, \bar{\mu}_{l}\right]$ we will always have the same expression for the value function $\tilde{\varphi}(\mu)$, or the same behavior given by the efficient extreme point $Q_{l}=\left(z_{l, \text { cost }}, z_{l, \text { phosphorus excretion }}\right)$, and the change in the behavior will happend only when the taxation level $\mu$ passes through the extremities $\underline{\mu}_{l}$ or $\bar{\mu}_{l}$ of this interval.

These problems of taxation are nice examples of abrupt (discrete) changes in behavior depending on the level of taxation of one criterion.

\subsubsection{The Two Kinds of Excretion as Criteria}

As a curiosity, we have computed the Pareto set for the bicriteria problem where 
the two kinds of excretions are considered. This bicriteria problem is given by

$$
\left(P_{p r, p h}\right) \begin{cases} & \min z_{2}=q_{p r} x \\ & \min z_{3}=q_{p h} x \\ \text { subject to } & \\ & x \in \mathcal{S} .\end{cases}
$$

Table 5 presents the set of efficient extreme points of the Pareto set in the criteria space. Its corresponding Pareto curve is sketched in Figure 3. This table shows the opposite effect of trying to reduce simultaneously both excretions. Minimizing one excretion leads to an increse in the other excretion. For this problem, the algorithm detects $L=5$ segments and 6 extreme points. A total of 12 calls to the linear program solver was required (the predicted maximum is $2 L+3=13)$.

For each $l=0, \cdots, 5$, the value function is

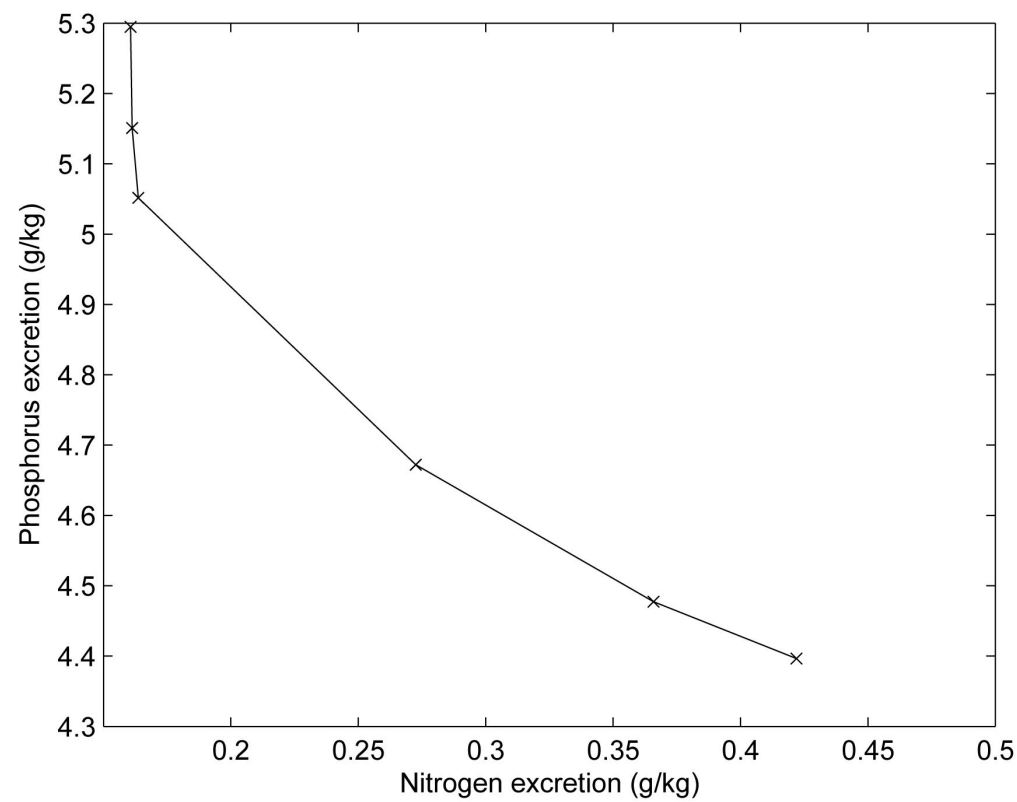

Figure 3. Pareto curve: phosphorus excretion vs nitrogen excretion.

Table 5. Efficient extreme points in the criterion space $\mathbb{R}^{2}$ for $\mathbb{R}^{2}$ pour $(P(p r, p h))$.

\begin{tabular}{cccccc}
\hline \multicolumn{5}{c}{ Pareto set } \\
\hline 1 & $\underline{\lambda}_{l}$ & $\bar{\lambda}_{l}$ & $\begin{array}{c}z_{l, \text { nitrogen excretion }} \\
\mathrm{g} / \mathrm{kg}\end{array}$ & $\begin{array}{c}z_{l, \text { phosphors excertion }} \\
\mathrm{g} / \mathrm{kg}\end{array}$ & $\begin{array}{c}z_{l, \text { cost }} \\
\$ / \mathrm{kg}\end{array}$ \\
\hline 0 & 0 & 0.00410 & 0.16066 & 5.29463 & 0.42713 \\
1 & 0.00410 & 0.02350 & 0.16125 & 5.15082 & 0.44249 \\
2 & 0.02350 & 0.22291 & 0.16364 & 5.05164 & 0.43631 \\
3 & 0.22291 & 0.32435 & 0.27253 & 4.67205 & 6.65922 \\
4 & 0.32435 & 0.40870 & 0.36591 & 4.47753 & 12.20160 \\
5 & 0.40870 & 1.00000 & 0.42199 & 4.39641 & 15.32799 \\
\hline
\end{tabular}




$$
\varphi(\lambda)=(1-\lambda) z_{l, \text { nitrogen excretion }}+\lambda z_{l, \text { phosphorus excretion }}
$$

for $\lambda \in\left[\underline{\lambda}_{l}, \bar{\lambda}_{l}\right]$. For all value of the parameter $\lambda$ in the interval $\left[\underline{\lambda}_{l}, \bar{\lambda}_{l}\right]$ we will have the same expression for the value functionn $\varphi(\lambda)$ or the same behavior $\left(z_{l \text {,nitrogen excretion }}, z_{l \text {,phosphorus excretion }}\right)$ and a change in the behavior will happend for values of the parameter $\lambda$ corresponding to the extremities $\underline{\lambda}_{l}$ ou $\bar{\lambda}_{l}$ of this interval.

Let us observe that the last line of Table $3(l=10)$ corresponds to the first line of Table $5(l=0)$ and the last line of Table $4(l=22)$ corresponds to the last line of Table $5(l=5)$.

\section{Conclusion}

In this paper we have considered bicriteria linear programming problems and have presented an elementary and efficient algorithm to compute the Pareto set in the criterion space. We have illustrated the method on a real important application. This application also suggests that it could be interresting to extend the method to three-criteria problems. Moreover it could be interesting to compare our method to other methods to find the Pareto set in the criterion space, but it is out of the scope of this paper and could be a nice subject for a future research.

\section{Acknowledgements}

This work has been supported in part by the Natural Sciences and Engineering Research Council of Canada and by the canadian corporation Swine Innovation Porc.

\section{Conflicts of Interest}

The authors declare no conflicts of interest regarding the publication of this paper.

\section{References}

[1] Dubeau, F., Julien, P.-O. and Pomar, C. (2011) Formulating Diets for Growing Pigs: Economic and Environmental Considerations. Annals of Operations Research, 190, 239-269. https://doi.org/10.1007/s10479-009-0633-1

[2] Adulbhan, P. and Tabucanon, M.T. (1977) Bicriterion Linear Programming. Computers \& Operations Research, 4, 147-153. https://doi.org/10.1016/0305-0548(77)90036-3

[3] Benson, H.P. (1979) Vector Maximization with Two Objective Functions. Journal of Optimization Theory and Applications, 28, 253-258. https://doi.org/10.1007/BF00933245

[4] Cohon, J.L., Church, R.L. and Sheer, D.P. (1979) Generating Multiobjective Trade-Offs. Water Resources Research, 15, 1001-1010. https://doi.org/10.1029/WR015i005p01001

[5] Gearhart, W.B. (1979) On the Characterization of Pareto-Optimal Solutions in Bicriteria Optimization. Journal of Optimization Theory and Applications, 27, 
301-307. https://doi.org/10.1007/BF00933233

[6] Geoffrion, A.M. (1967) Solving Bicriterion Mathematical Programs. Operations Research, 15, 39-54. https://doi.org/10.1287/opre.15.1.39

[7] Kiziltan, G. and Yucaoglu, E. (1982) An Algorithm for Bicriterion Linear Programming. European Journal of Operationl Research, 10, 406-411. https://doi.org/10.1016/0377-2217(82)90091-1

[8] Prasad, S.Y. and Karwan, M.H. (1992) A Note on Solving Bicriteria Linear Programming Problems Using Single Criteria Software. Computers \& Operations Research, 19, 169-173. https://doi.org/10.1016/0305-0548(92)90090-R

[9] Sadagan, S. and Ravindran, A. (1982) Interactive Solution of Bicriteria Mathematical Programs. Naval Research Logistics Quarterly, 29, 443-459. https://doi.org/10.1002/nav.3800290307

[10] Walker, J. (1978) An Interactive Method as an Aid in Solving Bicriteria Mathematical Programming Problems. Journal of the Operational Research Society, 29, 915-922. https://doi.org/10.1057/jors.1978.195

[11] Steuer, R.E. (1986) Multiple Criteria Optimization. Wiley, New York.

[12] Dubeau, F. and Kadri, A. (2012) Computation and Visualization of the Pareto Set in the Criterion Space for the Bicriteria Linear Programming Problem. International Journal of Mathematics and Computation, 15, 1-15.

[13] Benson, H.P. (1997) Generating the Efficient Outcome Set in Multiple Objective Linear Programs: The Bi-Criteria Case. Acta Mathematica Vietnamica, 22, 29-51.

[14] Bertsimas, D. and Tsitsiklis, J.N. (1997) Introduction to Linear Optimization. Athenas Scientific and Dynamic Ideas, Belmont.

[15] Murty, K.G. (1983) Linear Programming. Wiley, New York.

[16] Chvatal, V. (1983) Linear Programming. W.H. Freeman and Company, New York.

[17] Ferris, M.C., Mangasarian, O.L. and Wright, S.J. (2007) Linear Programming with MATLAB, MPS-SIAM Series on Optimization, Philadelphia.

[18] Stigler, G.J. (1945) The Cost of Subsistance. Journal of Farm Economics, 27, 303-314. https://doi.org/10.2307/1231810

[19] Dantzig, G.B. (1963) Linear Programming and Extensions. Princeton Press, Princeton.

[20] Dantzig, G.B. (1990) The Diet Problem. Interfaces, 20, 43-47. https://doi.org/10.1287/inte.20.4.43

[21] Garille, S.G. and Gass, S.I. (2001) Stigler's Diet Problem Revisited. Operations Research, 49, 1-13. https://doi.org/10.1287/opre.49.1.1.11187 